\title{
Cluster Structures, Halos, Skins, and S-Factors in Fermionic Molecular Dynamics
}

\author{
H. Feldmeier*, T. Neff ${ }^{\dagger}$ and R. Roth** \\ ${ }^{*}$ Gesellschaft für Schwerionenforschung, Planckstraße 1, 64291 Darmstadt, Germany \\ ${ }^{\dagger}$ NSCL, Michigan State University, 1 Cyclotron, East Lansing, Michigan 48824-1321, USA \\ ** Institut für Kernphysik, TU Darmstadt, Schlossgartenstraße 9, 64289 Darmstadt, Germany
}

\begin{abstract}
Light nuclei are studied in the Fermionic Molecular Dynamics model. No a priori assumptions are made with respect to cluster structure or single-particle energies. The same effective interaction based on the Argonne V18 interaction is used for all nuclei. Short-range central and tensor correlations are treated explicitly using a unitary correlation operator. Calculations of binding energies and radii for Helium and Carbon isotopes are presented. The evolution of cluster structures and halos with increasing neutron number is discussed. The spectrum of ${ }^{12} \mathrm{C}$ is calculated in a multiconfiguration calculation. The molecular structure of the excited states is investigated. The astrophysical S-factors are calculated for the fusion of different Oxygen isotopes.
\end{abstract}

Keywords: Fermionic Molecular Dynamics, nuclear structure, halo nuclei, S-factor PACS: 21.10.Gv, 21.60.Gx, 25.60.-t, 25.60.Pj, 24.10.Cn

\section{FERMIONIC MOLECULAR DYNAMICS}

The $A$-body basis states in Fermionic Molecular Dynamics (FMD) [1] are parity and angular momentum projected Slater determinants $|Q\rangle$

$$
\left|Q_{M K}^{J^{\pi}}\right\rangle=P_{M K}^{J^{\pi}}|Q\rangle=P_{M K}^{J}\left|Q^{\pi}\right\rangle=P_{M K}^{J}\left(|Q\rangle+(-1)^{\pi} \Pi|Q\rangle\right)
$$

of single particle states $\left|q_{i}\right\rangle$

$$
|Q\rangle=\mathscr{A}\left\{\left|q_{1}\right\rangle \otimes \ldots \otimes\left|q_{A}\right\rangle\right\}
$$

The single-particle wave functions are described by Gaussian wave packets which are localized in phase-space

$$
\langle\mathbf{x} \mid q\rangle=\sum_{i} c_{i} \exp \left\{-\frac{\left(\mathbf{x}-\mathbf{b}_{i}\right)^{2}}{2 a_{i}}\right\}\left|\chi_{i}\right\rangle \otimes|\xi\rangle .
$$

FMD treats the set $q$ consisting of the complex parameters: $a_{i}$ (width), $\mathbf{b}_{i}$ (position in coordinate and momentum space), $\chi_{i}$ (spin direction) and $c_{i}$ as variational parameters that can be different for each wave packet. A superposition of two Gaussian wave packets is used for each single-particle state $\left|q_{k}\right\rangle$ in order to improve the representation of the surface. $\xi$ stands for proton or neutron. The AMD approach [2] is a model very similar to FMD but the width parameter $a$ is common to all wave packets and the spins are either "up" or "down". 
As a first step FMD many-particle states are determined by minimizing the intrinsic energy of parity projected Slater determinants

$$
E\left[\left|Q^{\pi}\right\rangle\right]=\frac{\left\langle Q^{\pi}\left|H_{e f f}-T_{c m}\right| Q^{\pi}\right\rangle}{\left\langle Q^{\pi} \mid Q^{\pi}\right\rangle}
$$

with respect to the parameters of all single-particle states. After the minimization the many-particle state is projected on angular momentum. The correlation energy obtained by the projection can be very large for the often deformed and clustered nuclei in the $p$-shell. We therefore improve this projection after variation procedure $\left(\mathrm{PAV}^{\pi}\right)$ by implementing a variation after projection (VAP) procedure in the spirit of the generator coordinate method (GCM). We minimize the energy of the Slater determinants under additional constraints on collective variables such as: radius, dipole, quadrupole or octupole moment. The VAP minimum can then be found by minimizing the projected energies with respect to the constraints. A further improvement is achieved by diagonalising the Hamiltonian in a set of many-body states. This also allows for the study of excited states.

\section{EFFECTIVE INTERACTION}

For our calculations we use an effective interaction that is derived from the realistic Argonne V18 interaction by means of the Unitary Correlation Operator Method (UCOM) $[3,4,5,6]$. The correlated interaction includes the short-range central and tensor correlations induced by the repulsive core and the tensor force. The correlated interaction no longer connects to high momenta and can be used directly with the simple manybody states of a Hartree-Fock or FMD approach. A two-body correction term is added which consists of a central momentum dependent part that is adjusted to fix the saturation properties by fitting to the binding energies and radii of ${ }^{4} \mathrm{He},{ }^{16} \mathrm{O},{ }^{40} \mathrm{Ca}$ and an isospin dependent spin-orbit term that is fitted to the binding energies of ${ }^{24} \mathrm{O},{ }^{34} \mathrm{Si}$ and ${ }^{48} \mathrm{Ca}$. It is supposed to correct for missing contributions from three-body correlations and genuine three-body forces as well as other long range correlations not represented well by the simplified many-body Hilbert space. The correction term used in this paper differs slightly from the one in [5] as ${ }^{16} \mathrm{O}$ and ${ }^{40} \mathrm{Ca}$ are considered as tetrahedral $\alpha$-cluster states. These are about $5 \mathrm{MeV}$ lower in energy after angular momentum projection than the spherical trial states. In total the correction term contributes about $15 \%$ of the potential energy.

\section{HELIUM ISOTOPES}

Fig. 1 shows the intrinsic states obtained by minimizing the energy of the Helium isotopes with parity projected states. In all nuclei a dipole deformation caused by a displacement of the neutrons against the $\alpha$-core is found. In ${ }^{6} \mathrm{He}$ the configuration with two neutrons on the same side of the core is preferred to configurations with the two neutrons located on opposite sides of the core. In ${ }^{8} \mathrm{He}$ one approaches the $p_{3 / 2}$ neutron 

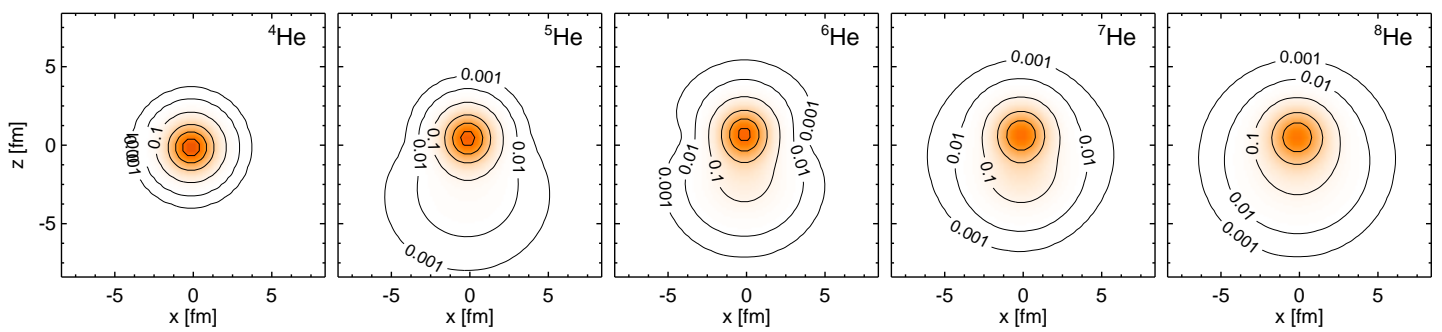

FIGURE 1. Intrinsic shapes of Helium isotopes corresponding to the variation after parity projection minima. We show cuts through the nucleon density calculated with the intrinsic state before parity projection. Densities are given in units of nuclear matter density $\rho_{0}=0.17 \mathrm{fm}^{-3}$.

shell closure with an almost spherical neutron distribution. In Fig. 3 the binding energies and matter radii obtained after angular momentum projection $\left(\mathrm{PAV}^{\pi}\right)$ are compared to the experimental binding energies and radii. To improve the many-body states we create additional configurations using the dipole moment as a generator coordinate. The multiconfiguration calculations reproduce the experimental binding energies and radii very well. This illustrates the importance of the soft-dipole mode, which is realized in the form of ground state correlations, for the understanding of the borromean nature of ${ }^{6} \mathrm{He}$ and ${ }^{8} \mathrm{He}$. Besides the neutron halo, in Fig. 2 we can see the broadened proton distribution that is caused by the motion of the $\alpha$-core against the center of mass of the nucleus. For ${ }^{6} \mathrm{He}$ we calculate a charge radius of $2.02 \mathrm{fm}$ that has to be compared to the recently measured value of $2.054 \pm 0.014 \mathrm{fm}[8]$.

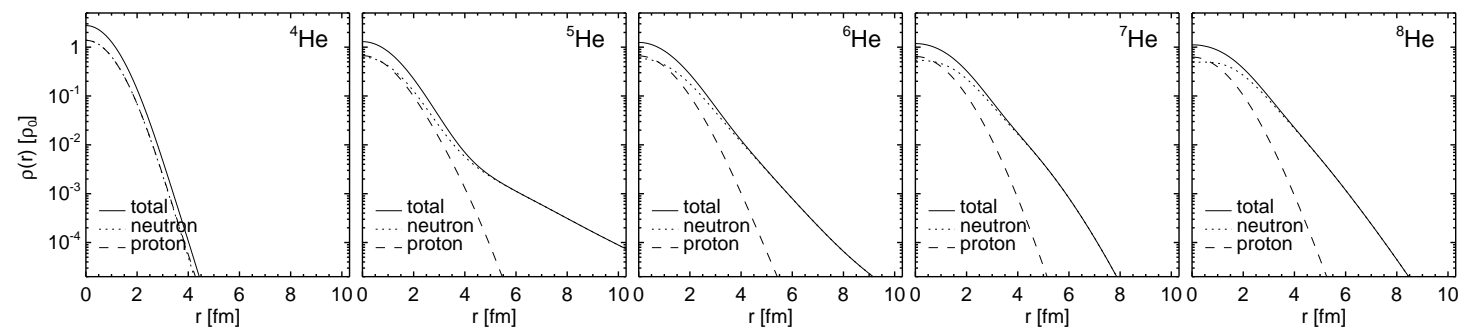

FIGURE 2. Radial density distributions for the Helium isotopes using angular momentum projected multiconfiguration states. The center of mass motion has been removed.

\section{CARBON ISOTOPES}

In the Carbon isotopes we observe many different structures as can be seen in Fig. 4. We see a prolate ${ }^{10} \mathrm{C}$ and find oblate triangular structures in ${ }^{11} \mathrm{C},{ }^{12} \mathrm{C}$ and ${ }^{13} \mathrm{C}$. In ${ }^{14} \mathrm{C}$ we find that the fully occupied neutron $p$-shell induces a spherical configuration. In ${ }^{15} \mathrm{C}$ the additional neutron occupies mainly a $1 s_{1 / 2}$ state whereas the subsequent isotopes from ${ }^{16} \mathrm{C}$ to ${ }^{18} \mathrm{C}$ show prolate deformations caused by filling up neutrons in the $s d$ shell. We also observe that the deformation is dominated by the neutrons. In Fig. 5 the proton and neutron densities of the intrinsic state of ${ }^{16} \mathrm{C}$ are shown. If we project out from this intrinsic state the $0^{+}$and $2^{+}$states and calculate the $B(E 2)$ value for the 


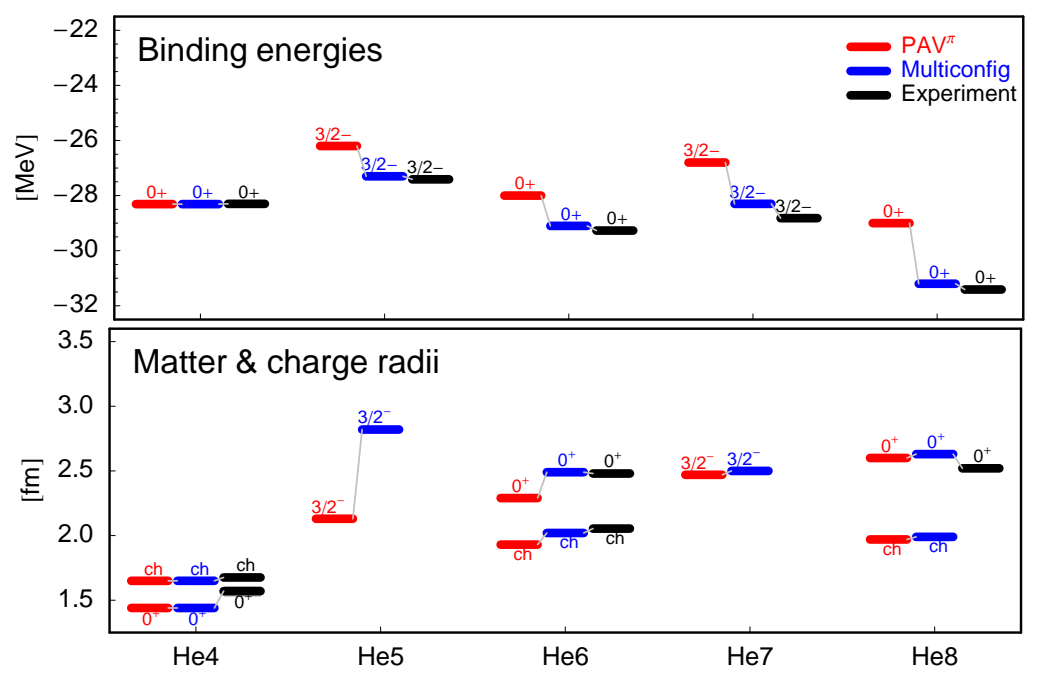

FIGURE 3. Binding energies and matter and charge radii for the Helium isotopes. Charge radii are indicated by ch. Results are given for the $\mathrm{PAV}^{\pi}$ and the multiconfiguration calculations. Experimental matter radii are taken from [7]. The experimental charge radius of ${ }^{6} \mathrm{He}$ is given in [8].

$0^{+} \rightarrow 2^{+}$transition we obtain a value of $9.3 e^{2} \mathrm{fm}^{4}$, which should be compared to the surprisingly small experimental value of $3.15 \pm 0.95 e^{2} \mathrm{fm}^{4}$ [9]. No effective charges are used for protons and neutrons, hence the electrical quadrupole moment reflects directly the intrinsic shape of the proton distribution. In our picture the much smaller than expected $B(E 2)$ value is caused by an almost spherical proton distribution which is decoupled from the deformed neutron distribution (see Fig. 5).

In Fig. 6 we compare calculated values with experimental ones for binding energies and radii. We find very good agreement between the multiconfiguration calculations and the experimental binding energies. The binding energies obtained for the single Slater determinants of the $\mathrm{PAV}^{\pi}$ approach are able to reproduce the evolution of the binding

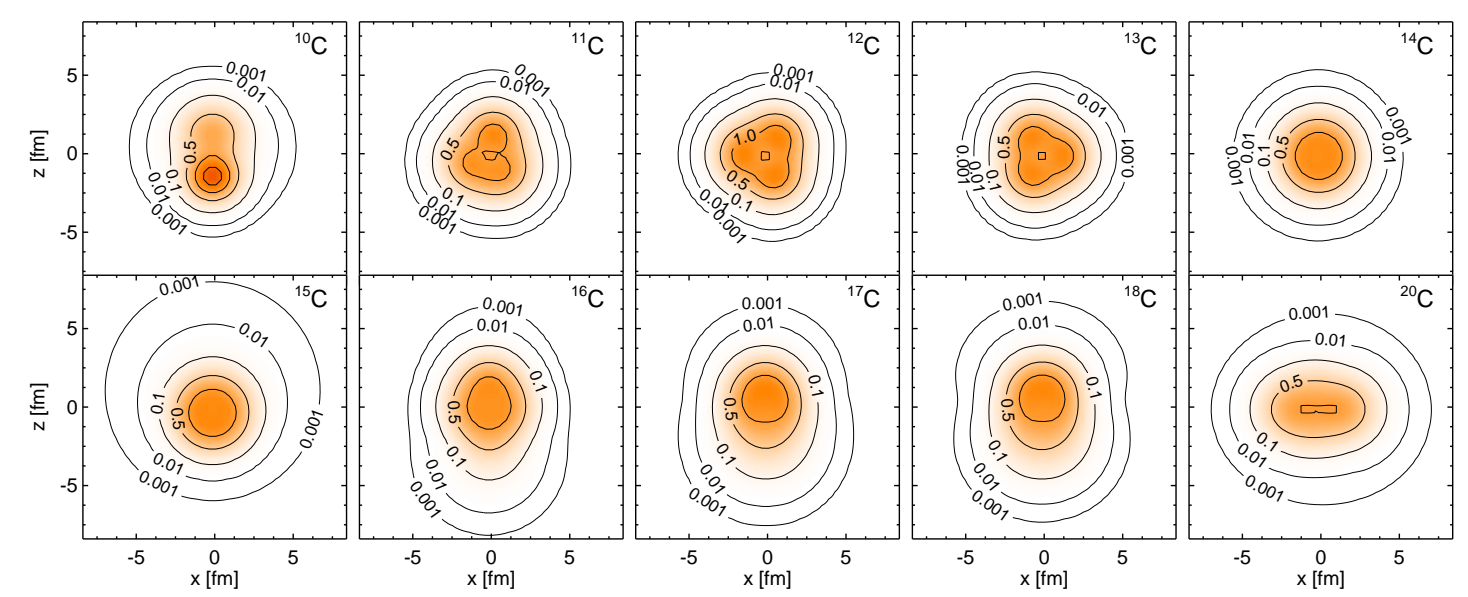

FIGURE 4. Intrinsic densities of Carbon isotopes corresponding to the variation after parity projection minima. 


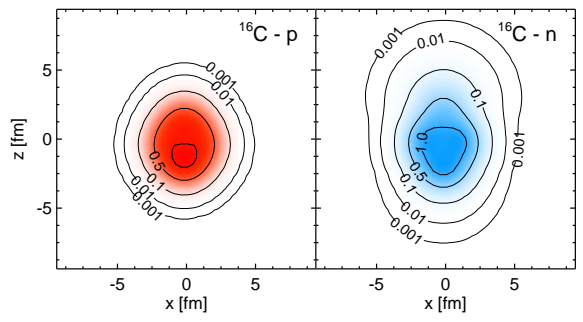

FIGURE 5. Proton (left) and neutron (right) densities of the ${ }^{16} \mathrm{C}$ intrinsic state for the $\mathrm{PAV}^{\pi}$ minimum.

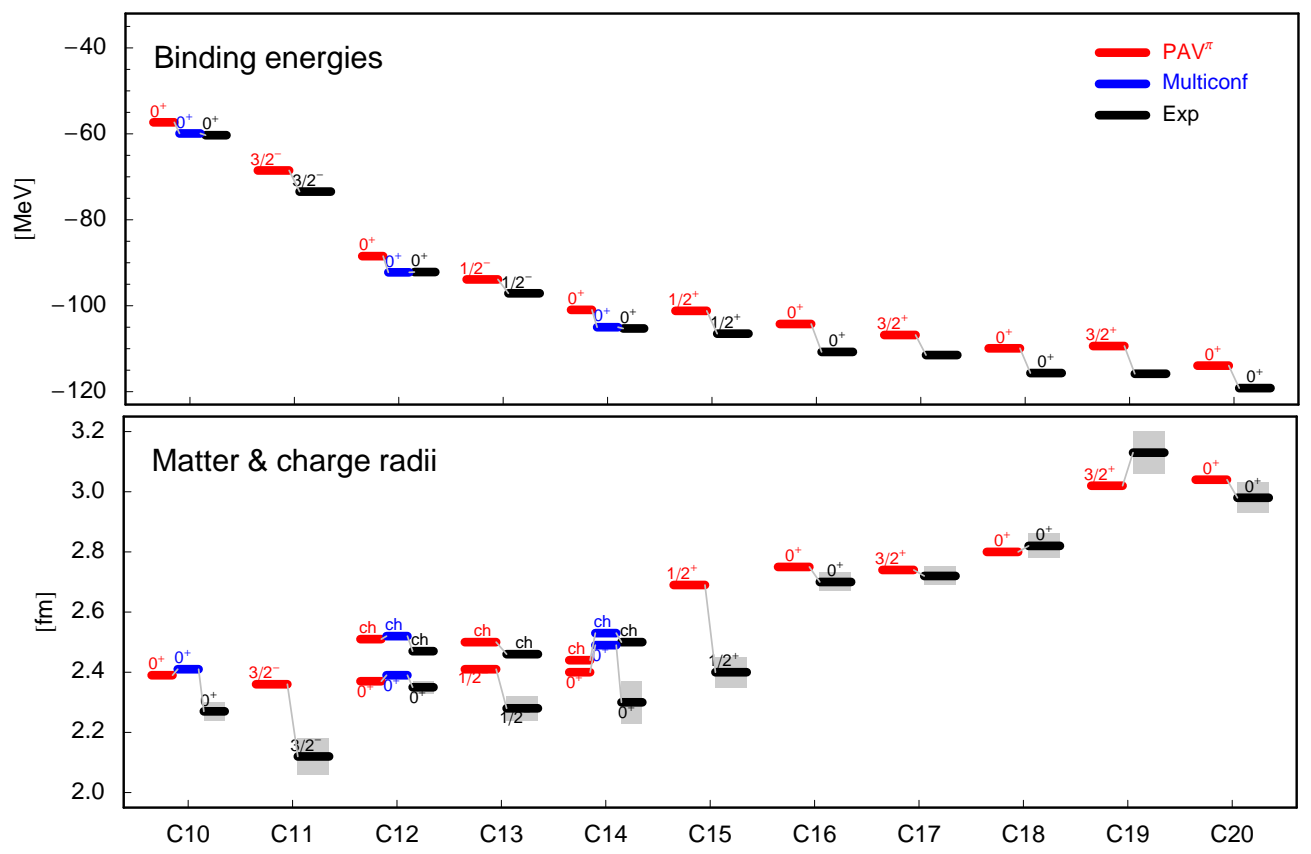

FIGURE 6. Energies and matter and charge radii of Carbon Isotopes. Calculated charge radii (indicated by $\mathrm{ch}$ ) are only shown if experimental values are known. Multiconfiguration calculations have only been done for ${ }^{10} \mathrm{C},{ }^{12} \mathrm{C}$ and ${ }^{14} \mathrm{C}$.

energies up to the heavy isotopes. For ${ }^{12} \mathrm{C}$ to ${ }^{14} \mathrm{C}$ experimental charge radii are known and reproduced well by our calculations. For the matter radii we find significantly larger values for the lighter isotopes up to ${ }^{14} \mathrm{C}$. There is some uncertainty in the experimental determination of the matter radii beyond the given error bars and as we find a good agreement for our calculated charge radii in this region there might be a problem with these experimental results. In case of ${ }^{15} \mathrm{C}$ our matter radius is much larger. This is probably explained by the very weak binding of the $1 s_{1 / 2}$ neutron. Our calculated neutron separation energy of ${ }^{15} \mathrm{C}$ is too small, the $s$-orbit is therefore much more extended. For the heavier isotopes the calculated matter radii agree well with experimental values. 
TABLE 1. Energies, radii and $B(E 2)$ transition strength calculated with various FMD states.

\begin{tabular}{lrrr}
\hline & $\mathbf{E}[\mathrm{MeV}]$ & $\mathbf{r}_{\text {charge }}[\mathrm{fm}]$ & $\mathbf{B}(\mathbf{E 2})\left[e^{2} \mathrm{fm}^{4}\right]$ \\
\hline V/PAV & -81.4 & 2.36 & - \\
VAP $\alpha$-cluster & -79.1 & 2.70 & 76.9 \\
PAV ${ }^{\pi}$ & -88.5 & 2.51 & 36.3 \\
VAP & -89.2 & 2.42 & 26.8 \\
Multiconfig (4) & -92.2 & 2.52 & 42.8 \\
Multiconfig (14) & -92.4 & 2.52 & 42.9 \\
\hline Experiment & -92.2 & 2.47 & $39.7 \pm 3.3$ \\
\hline
\end{tabular}

\section{${ }^{12}$ C SPECTRUM}

The structure of ${ }^{12} \mathrm{C}$ is characterized by an interplay between shell-model and cluster structure. If we perform an unconstrained minimization of the energy we end up with a spherical ${ }^{12} \mathrm{C}$ that is identical to the $\left(0 s_{1 / 2}\right)^{4}\left(0 p_{3 / 2}\right)^{8}$ shell model configuration. The energy for this configuration is about $10 \mathrm{MeV}$ too high compared to experiment and the radius is too small (see Tab. 1). If we try a Brink type $\alpha$-cluster wave function we find a configuration that has a similar energy but a radius much larger than experiment. Whereas in the shell model configuration the spin-orbit interaction contributes strongly to the binding energy, the $\alpha$-cluster configuration has no contribution from the spinorbit force. The $\alpha$-cluster configuration on the other hand has a very big correlation energy due to the angular momentum projection. If we minimize after parity projection $\left(\mathrm{PAV}^{\pi}\right)$ or search for the variation after projection minimum (VAP using the radius and the octupole moment as constraints), we find solutions that are significantly lower in energy. In these configurations the spin-orbit and correlation energy contributions are somewhere in between the pure shell model and $\alpha$-cluster configurations.

The angular momentum projection of the $\mathrm{PAV}^{\pi}$ solution already provides a reasonable description of the ground state rotational band of ${ }^{12} \mathrm{C}$. We can improve this description by performing a multiconfiguration calculation with four intrinsic states. Starting with the VAP configuration we consecutively add configurations that improve the ground state energy. In the Multifonfig(4) calculation the ground state rotational band including the $B(E 2)$ transition strength for the $0^{+} \rightarrow 2^{+}$transition is well reproduced. In addition we find a second $0^{+}$state, the famous Hoyle state. A description of this state as a Bose
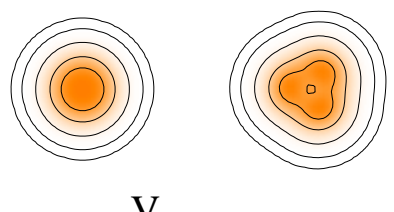

$\mathrm{V}$

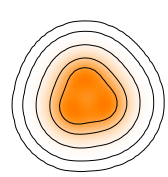

$\mathrm{PAV}^{\pi}$
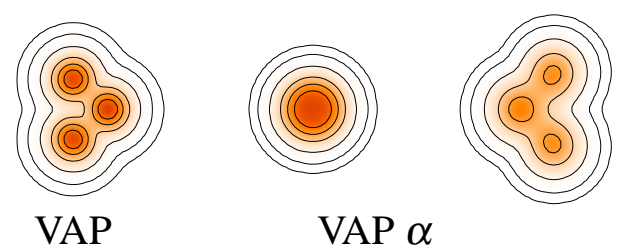

VAP $\alpha$

FIGURE 7. Intrinsic shapes of ${ }^{12} \mathrm{C}$ corresponding to the variation (V), the variation after parity projection $\left(\mathrm{PAV}^{\pi}\right)$, the variation after angular momentum projection (VAP) and the variation after projection for a Brink $\alpha$-cluster trial state (VAP $\alpha$ ). The four right most configurations are used for the Multiconfig(4) calculation. 


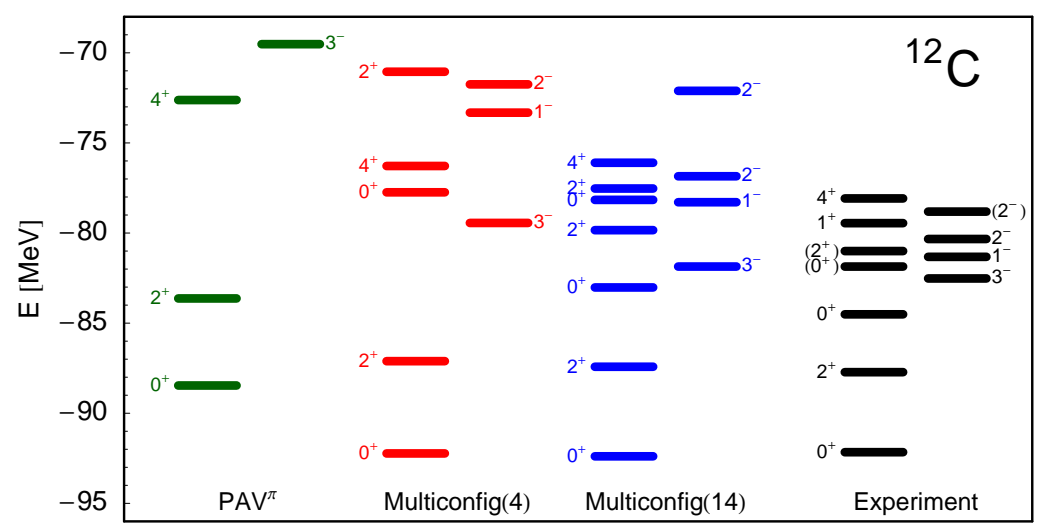

FIGURE 8. Spectrum of ${ }^{12} \mathrm{C}$ calculated using the single intrinsic state obtained in the variation after parity projection calculation $\left(\mathrm{PAV}^{\pi}\right)$ and in multiconfiguration calculations using 4 and 14 Slater determinants. The experimental spectrum is shown for comparison.

condensate of $\alpha$-particles has been proposed [11]. We improve our many-body basis by creating 10 additional $\alpha$-cluster configurations using quadrupole and octupole moments as constraints. The resulting Multiconfig(14) result is displayed in Fig. 8 indicating a significant improvement in the $0_{2}^{+}$energy. We obtain a monopole matrix element for the $0_{1}^{+} \rightarrow 0_{2}^{+}$transition of $5.67 \mathrm{fm}^{2}$, which should be compared with the experimental value of $(5.5 \pm 0.2) \mathrm{fm}^{2}$.

\section{ASTROPHYSICAL S-FACTOR}

By locating the FMD ground states of two nuclei on a grid at different distances and antisymmetrizing the product state, one can span a many-body Hilbert space that describes the relative motion of the nuclei. From the Hamiltonian matrix represented in this space one can determine the nucleus-nucleus potential. From that the probability
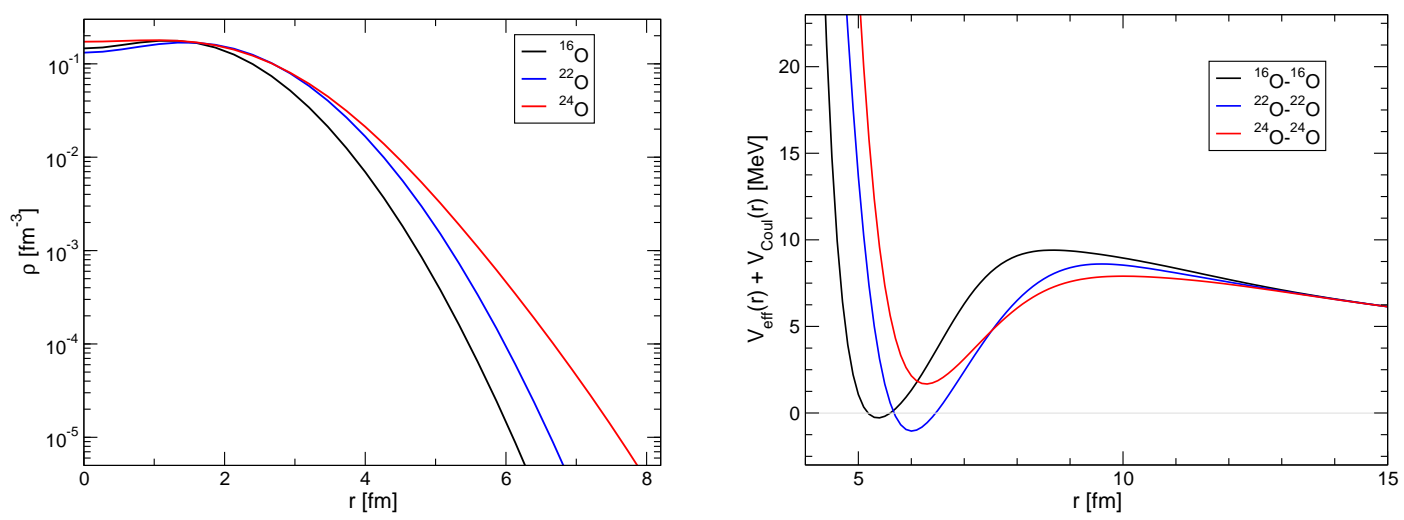

FIGURE 9. L.h.s.: mass densities (point nucleons) for different Oxygen isotopes. R.h.s.: nucleusnucleus potentials calculated in the frozen state approximation. 
for fusion can be calculated at energies much below the Coulomb barrier, as occurs in astrophysical scenarios. The fusion of neutron rich unstable isotopes is expected to take place in pycno-nuclear reactions when the density is larger than $10^{-12} \mathrm{~g} / \mathrm{cm}^{3}$ and nuclei are densely frozen in lattice positions, such that the Coulomb barrier is reduced by the small distances and the electron cloud between neighbouring nuclei [12]. In a microscopic model like FMD there are no adjustable parameters in calculating the potential, in particular, the isospin dependence comes from the isospin dependence of the nucleonnucleon interaction and the neutron to proton ratios in the surface of the nuclei. As an example, in Fig. 9 we display the ground state densities of ${ }^{16} \mathrm{O},{ }^{22} \mathrm{O}$, and ${ }^{24} \mathrm{O}$ together with the calculated nucleus-nucleus potential for ${ }^{16} \mathrm{O}+{ }^{16} \mathrm{O},{ }^{22} \mathrm{O}+{ }^{22} \mathrm{O}$ and ${ }^{24} \mathrm{O}+{ }^{24} \mathrm{O}$. From Fig. 10 one sees a dramatic increase in fusion probability for the neutron rich isotopes, both below and above the barrier (hinted at by the respective kink in the S-factor).

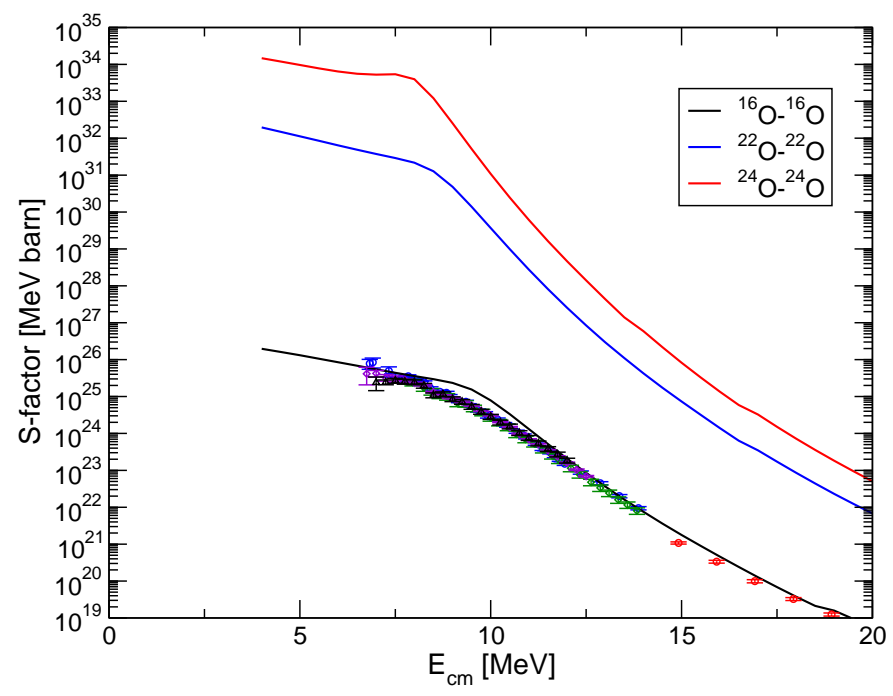

FIGURE 10. S-factor as function of relative energy for ${ }^{16} \mathrm{O}+{ }^{16} \mathrm{O},{ }^{22} \mathrm{O}+{ }^{22} \mathrm{O}$ and ${ }^{24} \mathrm{O}+{ }^{24} \mathrm{O}$. In the case of ${ }^{16} \mathrm{O}+{ }^{16} \mathrm{O}$ available data have been added.

\section{REFERENCES}

1. H. Feldmeier and J. Schnack, Rev. Mod. Phys. 72 (2000) 655. and references therein

2. Y. Kanada-En'yo and H. Horiuchi, Prog. Theor. Phys. Suppl. 142 (2001) 205

3. H. Feldmeier, T. Neff, R. Roth, J. Schnack, Nuc. Phys. A632 (1998) 61

4. T. Neff and H. Feldmeier, Nuc. Phys. A713 (2003) 311

5. R. Roth, T. Neff, H. Hergert, H. Feldmeier, Nuc. Phys. A745 (2004) 3

6. R. Roth, H. Hergert, P. Papakonstantinou, T. Neff, H. Feldmeier, accepted by Phys. Rev. C, xxxPreprint nucl-th/0505080

7. A. Ozawa, T. Suzuki, I. Tannihata, Nuc. Phys. A693 (2001) 32

8. L.-B. Wang et al, Phys. Rev. Lett 93 (2004) 142501

9. N. Imai et al, Phys. Rev. Lett. 92 (2004) 062501

10. S.K. Bogner, T.T.S. Kuo and A. Schwenk, Phys. Reports 386 (2003) 1

11. Y. Funaki, A. Tohsaki, H. Horiuchi, P. Schuck and G. Röpke, Phys. Rev. C67 (2003) 051306

12. L.R. Gasques, A.V. Afanasjev, E.F. Aguilera, M. Beard, L.C. Chamon, P. Ring, M. Wiescher, D.G. Yakovlev, Phys. Rev. C 72 (2005) 025806 